BMJ Open Sport \& Exercise Medicine

\section{Acute high-intensity aerobic exercise affects brain-derived neurotrophic factor in mild cognitive impairment: a randomised controlled study}

To cite: Devenney KE, Guinan EM, Kelly ÁM, et al. Acute high-intensity aerobic exercise affects brain-derived neurotrophic factor in mild cognitive impairment: a randomised controlled study. BMJ Open Sport \& Exercise Medicine 2019;5:e00499. doi:10.1136/ bmjsem-2018-000499

Accepted 16 May 2019
Check for updates

C A Author(s) (or their employer(s)) 2019. Re-use permitted under CC BY-NC. No commercial re-use. See rights and permissions. Published by BMJ.

${ }^{1}$ School of Medicine, Trinity College Dublin, University of Dublin, Dublin, Ireland ${ }^{2}$ Department of Physiology, Trinity College Institute of Neuroscience, Dublin, Ireland ${ }^{3}$ School of Computer Science and Statistics, Trinity College Dublin, Dublin, Ireland ${ }^{4}$ Geriatrics, Radboud UMC, Nijmegen, The Netherlands ${ }^{5}$ Institute of Movement and Neurosciences, German Sport University Cologne, Köln, Germany

Correspondence to Dr Kate E Devenney; devennek@tcd.ie

\section{ABSTRACT}

Objective To investigate the brain-derived neurotrophic factor (BDNF) and cognitive response to a short bout of high-intensity aerobic exercise in older adults with mild cognitive impairment (MCl).

Methods Participants were randomised to one of two testing schedules, completing either a standardised exercise test (group A) or a resting control condition (group B). Blood sampling and cognitive measures (visuospatial learning and memory, sustained attention and executive function) were collected at baseline (T1) and postintervention (T2). An additional measurement of study outcomes was collected after exercise (T3) in group B only. Results 64 participants (female $53.2 \%$, mean age $70.5 \pm 6.3$ years) with $\mathrm{MCl}$ were recruited. From $\mathrm{T} 1$ to $\mathrm{T} 2$, serum BDNF (sBDNF) concentration increased in group $A(n=35)$ (median (Md) 4564.61 IQR $5737.23 \mathrm{pg} / \mathrm{mL}$ to Md $5173.27 \pm 5997.54 \mathrm{pg} / \mathrm{mL}$ ) and decreased in group $B$ (Md 4593.74 $\pm 9558.29 \mathrm{pg} / \mathrm{mL}$ to Md 3974.66 \pm 3668.22 $\mathrm{pg} / \mathrm{mL}$ ) (between-group difference $\mathrm{p}=0.024$, effect size $r=0.3$ ). The control group made fewer errors on the sustained attention task compared with the exercise group $(p=0.025)$. Measures of visuospatial learning and memory or executive function did not change significantly between groups.

Conclusion This study is the first to show that a short bout of high-intensity aerobic exercise increases peripheral sBDNF in a population with $\mathrm{MCl}$. However, acute exercise did not improve cognitive performance.

\section{INTRODUCTION}

Mild cognitive impairment (MCI) is regarded as a clinical prodrome to Alzheimer's disease (AD) and other dementias, affecting approximately $15 \%-20 \%$ of people over the age of $65,{ }^{1}$ with $10 \%-15 \%$ of people with MCI progressing to dementia per year. ${ }^{2}$ With the ongoing challenges in developing disease-modifying drugs, research into non-pharmacological strategies such as exercise, to prevent dementia or treat cognitive decline has gained increasing importance. A

\section{What are the new findings?}

A short bout of high-intensity exercise increased serum brain-derived neurotrophic factor (sBDNF) in a mild cognitive impairment (MCl) cohort.

- While high-intensity interventions were feasible in this patient group, significant improvements were not observed in cognitive performance.

\section{How might it impact on clinical practice?}

Further research is needed to establish if acute exercises increase resting sBDNF concentration. However, exercise training may be a potential strategy to address the declining BDNF profile in an MC population.

recent update to the American Academy of Neurology practice guideline on MCI now includes a recommendation for regular exercise as part of overall disease management. ${ }^{3}$

A significant body of work has investigated the effects of acute exercise on cognition. Meta-analytical reviews report an overall small positive effect on cognitive performance following acute exercise (mean effect 0.10-0.20), especially in areas of prefrontal cortex-dependent cognition. ${ }^{4-6}$ However, the underlying neurobiological basis of these results has received less attention.

Brain-derived neurotrophic factor (BDNF), a member of the neurotrophin family, plays a predominant role in neuronal plasticity ${ }^{7}$ and has been shown to decrease progressively with age, ${ }^{89}$ with attenuated loss and a corresponding decrease in cognitive function in MCI cohorts compared with elderly controls. ${ }^{10-12}$ Studies in both animal models and human subjects have described a number of neurobiological changes after an acute bout of exercise ${ }^{13}$ and recent evidence 


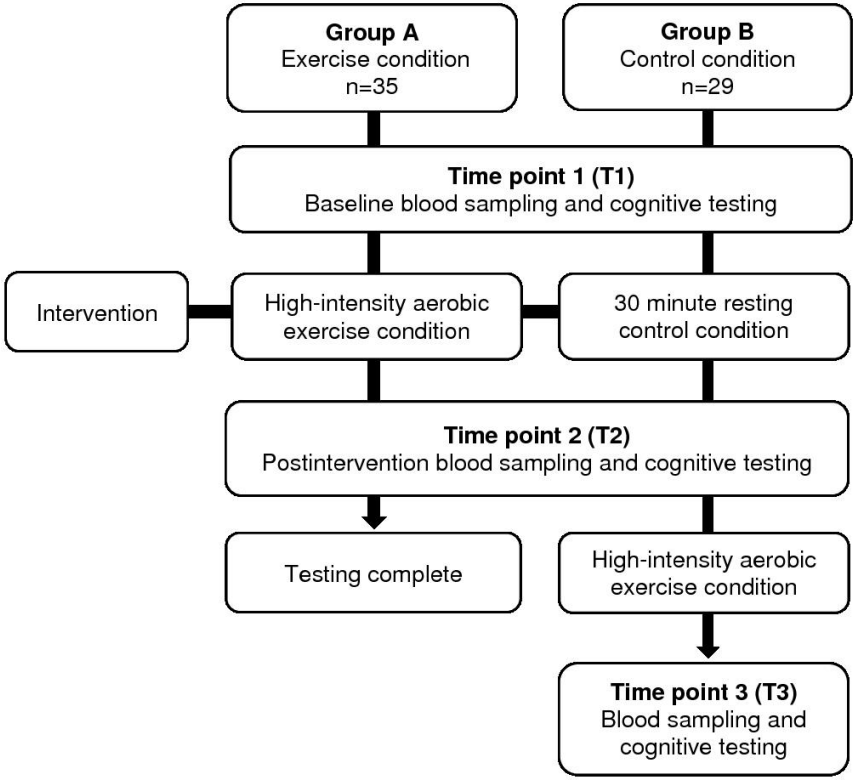

Figure 1 Study design and schedule of outcome assessments.

postulates BDNF as a mediator of the acute exercise-cognitive relationship in healthy cohorts. ${ }^{14}$

The transitional phase of MCI along the cognitive continuum presents an opportunity for targeted interventions such as the exercise-induced upregulation of BDNF, which may help slow the rate of cognitive decline. As chronic exercise is the cumulative result of regular bouts of acute exercise, understanding the neurobiological effects of varying acute exercise paradigms is an integral part of building a comprehensive understanding of the long-term effects of exercise on cognitive function. ${ }^{13}$ This information will help determine the optimal prescription of acute and chronic exercise and add to the emerging research that supports the role of exercise as a natural therapeutic tool to treat cognitive decline in older adults with MCI.

The aim of this study was to examine the effect of a short bout of high-intensity aerobic exercise on serum BDNF (sBDNF) and cognitive performance in individuals with MCI. The working hypothesis was that a short bout of high-intensity exercise would significantly increase sBDNF and cognitive performance compared with a resting control condition.

\section{METHODS}

\section{Study Design}

Participants were randomised to one of two testing schedules (figure 1) using a computer-generated randomisation list generated by a researcher independent of the study. Treatment allocation was concealed from the investigator. Group A represented the exercise condition while group B was designed to act as the control condition. Outcome measurements were collected at T1 (baseline) and T2 (after the exercise condition in group $\mathrm{A}$ and after a resting control condition for group B), with T3 measurements (after exercise) only collected in group B.

\section{Patient and Public Involvement (PPI)}

As no funds or time were allocared for PPI, participants were unable to be involved in study design stages. However, participants have been involved in the dissemination.

\section{Participants}

Inclusion and exclusion criteria

Participants were screened and recruited from a network of memory clinics $(n=38)$ and through community recruitment $(\mathrm{n}=26) .{ }^{15} 16$ Details of the study inclusion and exclusion criteria have been published in the NeuroExercise study protocol. ${ }^{16}$ To summarise, participants had a diagnosis of amnestic MCI due to $\mathrm{AD}$ according to the National Institute on Aging and Alzheimer's Association criteria, ${ }^{17}$ scored $18-26$ on the Montreal Cognitive Assessment (MoCA $)^{18}$ and were classified as having memory decline but not dementia (Clinical Dementia Rating global score $=0.5$ ). Participants met the following inclusion criteria: ECG without significant abnormalities, and medical clearance to undergo a symptom-limited cardiopulmonary exercise test (CPET). Participants were deemed ineligible if they had a diagnosis of $\mathrm{AD}$ or other type of dementia; any major psychiatric disorder; unstable cardiac, renal, lung, liver or other severe chronic disease; or engagement in moderate-intensity aerobic exercise training for more than $30 \mathrm{~min}$, three times per week, during the past 2 years.

\section{Study outcomes}

Data collection and study interventions were conducted at the Wellcome Trust-Health Research Board Clinical Research Facility in Dublin. Ethical approval was granted from Tallaght Hospital and St James' Hospital Joint Research and Ethics Committee (REC) and the RECs of referring sites. Due to limited study personnel, the outcome assessor was not blinded to intervention group. All study outcomes were collected in a non-fasting state between the hours of 09:00 and 15:00.

\section{Blood sampling}

Participants completed a 10 min resting period prior to baseline blood sampling (T1), with postintervention blood samples collected within a $10 \mathrm{~min}$ period following each experimental condition. Serum collection tubes were inverted five times and allowed to coagulate at room temperature before being centrifuged at $1300 \times g$ for 10 $\min$ at $4^{\circ} \mathrm{C}$. The resulting serum was removed, aliquoted and stored at $-80^{\circ} \mathrm{C}$ pending laboratory analysis. Samples and standards were analysed using commercially available BDNF DuoSet ELISA development systems kit (R\&D Systems Europe, Oxon, UK) in accordance with the manufacturer's instruction. Standard curve was constructed and concentration of BDNF in samples was calculated by extrapolation from these curves with results expressed in $\mathrm{pg} / \mathrm{mL}$. 
Cognitive performance

Cognitive tests were administered as per standardised test instructions and included the Cambridge Neuropsychological Test Automated Battery (CANTAB) Paired Associates Learning (PAL) task, a measure of visuospatial learning and memory which was considered the primary cognitive outcome. CANTAB PAL is regarded as a visuospatial memory task, highly sensitive to the memory impairments characteristic in prodromal $\mathrm{AD}$ that assesses visual memory and new learning through the testing of conditional learning of pattern-location associations. ${ }^{19}$ The Sustained Attention to Response Task (SART), a computerised Go-No-Go paradigm measuring sustained attention, and the Stroop Neuropsychological Screening Test (Stroop) measuring executive function were measured as secondary cognitive outcomes. Testing took approximately $30 \mathrm{~min}$ to complete.

\section{Intervention}

Participants performed a graded CPET to volitional exhaustion as per WHO protocol. ${ }^{20}$ The test was performed on a standard COSMED cycle ergometer with oxygen consumption $\left(\mathrm{VO}_{\mathrm{o}}\right)$ measured using the $\mathrm{K} 4 \mathrm{~B} 2^{2}$ equipment (K4B2 ${ }^{2}$ User Manual, COSMED, Italy). ECG and blood pressure measurements were collected at rest and for the duration of the test. The protocol commenced with a 3 min warm-up of unloaded cycling followed by an incremental phase of cycling exercise during which the load increased by $25 \mathrm{~W}$ every 2 min until the test was terminated and a 3 min cool down period was performed. The test was terminated when three of the following criteria were met: clinical signs of exhaustion, respiratory quotient $\geq 1.10$, reaching within 10 beats of the predicted heart rate max (220 age) or flattening of the $\mathrm{VO}_{2}$ uptake curve $(\leq 110 \mathrm{~mL}$ increase during the last minute). The control condition in group B consisted of 30 min lying in a semirecumbent position.

\section{Sample size}

A recent meta-analytical review reported that an acute bout of exercise increased BDNF levels with a moderate effect size (Hedges' $\mathrm{g}=0.46, \mathrm{SE}=0.08,95 \%$ CI 0.29 to 0.62 , $\mathrm{z}=5.49, \mathrm{p}<0.001) .{ }^{21}$ Using these values, with 0.05 significance level and $80 \%$ power, 32 participants were required per arm to demonstrate an effect size of 0.46 in people with MCI.

\section{Statistical analysis}

SPSS V.24 (SPSS) was used for analysis with statistical significance set at $\mathrm{p}<0.05$. Kolmogorov-Smirnov test $(\mathrm{p}>0.05)$ and visual inspection of histogram and $\mathrm{Q}-\mathrm{Q}$ plots were used to assess assumptions of normality on all baseline variables. In the case of non-normally distributed data, non-parametric tests were performed.

To standardise baseline data, BDNF data were examined for outliers, with measurements \pm 2 SDs removed from the mean excluded as per standard approach. ${ }^{22}$ Following these criteria, $\mathrm{n}=9$ were removed from further analysis. Second, relative change (\%) in BDNF was reported consistent with published reports. ${ }^{23} 24$

For the primary analysis of study outcomes, betweengroup differences between $\mathrm{T} 1$ and $\mathrm{T} 2$ were analysed using an independent samples t-test for normally distributed data and using a Mann-Whitney $U$ test for non-normally distributed data. In the secondary analysis, Friedman's test and analysis of variance were used to measure the change over time in study outcomes in group B. Wilcoxon signed-rank tests and paired sample t-tests were performed in post hoc analysis to determine the effect size and compare T1-T2, T2-T3 and T1-T3 in group B. As secondary analysis involved multiple comparisons, a Bonferroni correction was applied and alpha level was revised to 0.025 . An exploratory analysis of sex differences in BDNF and cognition was performed.

\section{RESULTS}

Participants were enrolled from 25 February 2016 and data collection for this study ended on 17 July 2017. Sixtyfour participants were enrolled and randomised to group A $(n=35)$ or group B $(n=29)$. Missing data included PAL at T1 (participant refusal to complete $\mathrm{n}=1$ ), SART at T1 (measurement error $\mathrm{n}=1$ ), missed venepuncture at $\mathrm{T} 2$ $(\mathrm{n}=1)$ and invalid Stroop T1-T3 (colour blindness $\mathrm{n}=2$, incomplete record sheet $\mathrm{n}=2$ ). Missing data were treated as missing at random and each missing observation removed from analysis.

\section{Participant characteristics}

Participant characteristics (age, sex, education, MoCA and body mass index) were similar between groups at baseline (table 1). Participants had a mean age of $70.5( \pm 6.3)$ years, $53.2 \%$ female, mean MoCA score of $22.0( \pm 2.5)$ and the majority $(\mathrm{n}=38,59.4 \%)$ were highly educated (>13 years). Cardiorespiratory (CR) fitness levels categorised based on age and gender norms revealed a large proportion $(\mathrm{n}=54,84.4 \%)$ were categorised as very poor

Table 1 Participant demographics by study group

\begin{tabular}{llll}
\hline Variable & $\begin{array}{l}\text { Group A: } \\
\text { Exercise } \\
\mathbf{n = 3 5}\end{array}$ & $\begin{array}{l}\text { Group B: } \\
\text { Control } \\
\mathbf{n = 2 9}\end{array}$ & P value \\
\hline Gender (M/F) & $13 / 22$ & $17 / 12$ & 0.14 \\
Age (years) & $71.7(5.3)$ & $69.0(7.2)$ & 0.09 \\
Education (years) & $12.5(3.0)$ & $14.0(3.2)$ & 0.07 \\
MoCA & $21.6(2.5)$ & $22.4(2.6)$ & 0.24 \\
$\mathrm{BMI}\left(\mathrm{kg} / \mathrm{m}^{2}\right)$ & $27.44(4.49)$ & $26.14(4.21)$ & 0.23 \\
$\mathrm{VO}_{2}$ max $(\mathrm{mL} / \mathrm{kg} /$ & $17.77(5.15)$ & $21.35(4.85)$ & $0.002^{*}$ \\
$\mathrm{~min})$ & & & \\
\hline
\end{tabular}

Data are presented as mean \pm SD for normally distributed data and as median \pm IQR for non-normally distributed data. Note statistical significance.

${ }^{*} \mathrm{P}<0.05$.

$\mathrm{BDI}$, body mass index; MoCa, Montreal Cognitive Assessment; $\mathrm{VO}_{2}$, oxygen consumption. 
Table 2 Measurement parameters of exercise intensity and duration in the exercise condition for both groups

\begin{tabular}{lccl}
\hline & $\begin{array}{l}\text { Group A } \\
\mathbf{n = 3 5}\end{array}$ & $\begin{array}{l}\text { Group B } \\
\mathbf{n = 2 9}\end{array}$ & P value \\
\hline Time (min:s) & $\begin{array}{l}\text { 09:11 } \\
(03: 63)\end{array}$ & $\begin{array}{l}10: 25 \\
(02: 26)\end{array}$ & 0.11 \\
\hline Max watts $\left(\mathrm{W}_{\max }\right)$ & $100(50)$ & $100(50)$ & 0.34 \\
Max HR (HR & $118(20)$ & $124(21)$ & 0.27 \\
Peak lactate $(\mathrm{mmol} / \mathrm{L})$ & $5.0(4.8)$ & $4.4(2.7)$ & 0.16 \\
Lactate change (mmol/L) & $2.9(3.5)$ & $2.8(3.5)$ & 0.33 \\
Respiratory exchange & 1.06 & 1.06 & 0.95 \\
ratio (RER) & $(0.12)$ & $(0.07)$ & \\
\hline
\end{tabular}

$\left(<20\right.$ th percentile).$^{25}$ Despite randomisation, CR fitness calculated using results of the CPET was significantly higher in group B $(21.35 \pm 4.85 \mathrm{~mL} / \mathrm{kg} / \mathrm{min})$ compared with group A $(17.77 \pm 5.15 \mathrm{~mL} / \mathrm{kg} / \mathrm{min})$.

There were no significant between-group differences in baseline measures of cognitive performance or sBDNF. Baseline sBDNF did not correlate with MoCA $(\mathrm{r}=0.10, \mathrm{p}=0.46)$, Paired Associates Learning Total Errors Adjusted (PALTEA) $(\mathrm{r}=0.10, \mathrm{p}=0.44)$ or age $(\mathrm{r}=-0.02$, $\mathrm{p}=0.87$ ). Baseline and postexercise BDNF did not correlate with CR fitness $\left(\mathrm{VO}_{2} \max\right.$ ) (baseline $\mathrm{r}=-0.01$, $\mathrm{p}=0.92$; postexercise $\mathrm{r}=0.01, \mathrm{p}=0.89)$. Baseline $\mathrm{sBDNF}$ concentration for male (Md 3699.52 $\pm 6758.59 \mathrm{pg} / \mathrm{mL}^{1}$ ) and female $\left(\mathrm{Md} 5058.11 \pm 6280.08 \mathrm{pg} / \mathrm{mL}^{1}\right)$ participants was comparable $(p=0.224)$. Measurements of duration and intensity in the exercise condition did not show any significant between-group differences (table 2). There were no adverse events.

\section{Primary analysis}

Between-group difference in BDNF and cognitive performance sBDNF concentration increased in group A (exercise condition) from $\mathrm{T} 1$ ( $\mathrm{Md} 4564.61 \pm 5737.23 \mathrm{pg} / \mathrm{mL}^{1}$ ) to T2 (Md $5173.27 \pm 5997.54 \mathrm{pg} / \mathrm{mL}^{1}$ ) and decreased in group B (resting control condition) from T1 (Md $\left.4593.74 \pm 9558.29 \mathrm{pg} / \mathrm{mL}^{1}\right)$ to $\mathrm{T} 2(\mathrm{Md} 3974.66 \pm 3668.22$ $\left.\mathrm{pg} / \mathrm{mL}^{1}\right) \quad(\mathrm{p}=0.024$, Effect Size $(\mathrm{ES})=0.3)$ (figure 2). The median $\%$ relative change in $\mathrm{SBDNF}$ from $\mathrm{T} 1$ to $\mathrm{T} 2$ in response to exercise in group A was $7.07 \%( \pm 31.30 \%)$ and $-13.3 \%( \pm 22.49 \%)$ in response to a resting control condition in group $\mathrm{B}(\mathrm{p}=0.06)$.

Surprisingly, SART commission error rate increased in group A (Md 0.50, $\mathrm{n}=34)$ and decreased in group B (Md $-1.0, \mathrm{n}=29$ ) from $\mathrm{T} 1$ to $\mathrm{T} 2, \mathrm{U}=654.000, \mathrm{z}=2.240, \mathrm{r}=0.28$. An acute bout of high-intensity exercise did not significantly alter PALTEA, Stroop colour word score (CWS) or SART reaction time (RT) (table 3 ).

\section{Secondary analysis}

Change over time within group B

Repeated measures analysis within group B indicated a statistically significant difference in sBDNF across the three time points $(\mathrm{p}=0.005)$. $\mathrm{sBDNF}$

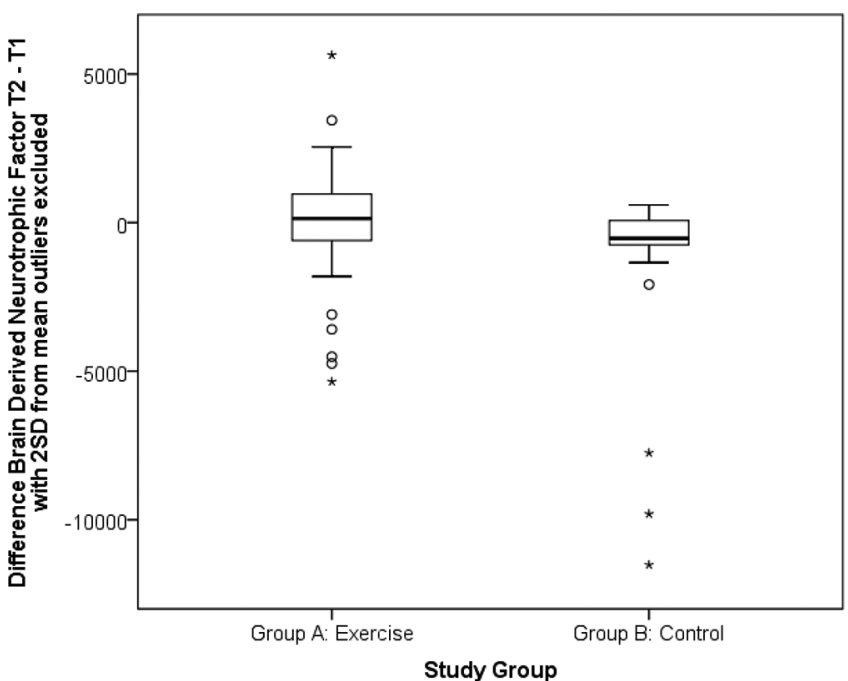

Figure 2 Boxplot showing between-group difference in serum BDNF (sBDNF) in response to a high-intensity exercise or resting control condition.

reduced significantly during the resting condition between $\mathrm{T} 1 \quad\left(\mathrm{Md}=4593.74 \pm 9558.29 \mathrm{pg} / \mathrm{mL}^{1}\right)$ and $\mathrm{T} 2$ $\left(\mathrm{Md}=3974.66 \pm 3668.22 \mathrm{pg} / \mathrm{mL}^{1}\right) \quad(\mathrm{p}=0.01, \mathrm{r}=0.38)$, and increased significantly from $\mathrm{T} 2 \quad(\mathrm{Md}=3974.66 \pm 3668.22$ $\mathrm{pg} / \mathrm{mL}^{1}$ ) to T3 following completion of a short bout of high-intensity aerobic exercise $(\mathrm{Md}=4790.90 \pm 6713.17$ $\left.\mathrm{pg} / \mathrm{mL}^{1}\right) \quad(\mathrm{p}=0.001, \mathrm{r}=0.48)$. The relative change in sBDNF is consistent with the findings of the primary analysis, with the mean $\%$ relative change in sBDNF concentration from T2 to T3 (postexercise) in group B of $15.7 \%( \pm 22.1 \%)$.

Stroop CWS (Wilks' lambda $=0.668, F(2,26)=6.461$, $\mathrm{p}=0.005$, multipartial $\left.\eta^{2}=0.33\right)$ and SART RT (2, $\mathrm{n}=29)=0.036, \quad \mathrm{p}=0.005$ ) changed significantly over time within group B. There were significant increases in Stroop CWS following participation in the exercise condition from $\mathrm{T} 1 \quad(\mathrm{M}=75.25 \pm 26.97)$ to $\mathrm{T} 3$ $(\mathrm{M}=83.93 \pm 21.47) \quad($ mean increase $=-8.67,95 \%$ CI -13.55 to -3.80$)(\mathrm{p}=0.001$, ES 0.34), indicating improved performance. SART RT reduced significantly in response to the control condition from T1 (Md $154.57 \mathrm{~ms} \pm 78.90$ $\mathrm{ms})$ to T2 (Md $123.61 \mathrm{~ms} \pm 79.62 \mathrm{~ms})(\mathrm{p}=0.004$, ES 0.37), with no significant change in SART commission errors. Additionally, PALTEA did not change significantly over time $(2, \mathrm{n}=29)=7.000, \mathrm{p}=0.03)$ although a non-significant decrease in error rate was observed from T1 (Md 44 \pm 22$)$ to T2 (Md 33 \pm 29$)(\mathrm{z}=-2.130, \mathrm{p}=0.03$, ES 0.27$)$ and from T1 to T3 $(\mathrm{Md}=28 \pm 30) \quad(\mathrm{z}=-2.232, \mathrm{p}=0.026$, ES 0.29). Results are presented in table 4 .

Sex differences in acute exercise BDNF and cognitive response There was a significant difference in SART RT pre-topost exercise in female participants (Pre Md 156.14 $\mathrm{ms} \pm 83.31 \mathrm{~ms}$ to post $\mathrm{Md} 134.62 \mathrm{~ms} \pm 69.86 \mathrm{~ms}$ ) compared with male participants (Pre Md $130.08 \mathrm{~ms} \pm 76.45 \mathrm{~ms}$ to post $\mathrm{Md} 136.77 \mathrm{~ms} \pm 67.56 \mathrm{~ms})(\mathrm{p}=0.025$, ES 0.28). There were no significant differences between male and female 
Table 3 Effect of exercise on BDNF and cognitive outcomes

\begin{tabular}{|c|c|c|c|c|c|}
\hline Variables & Baseline (T1) & Follow-up (T2) & $\begin{array}{l}\text { Change within } \\
\text { group }\end{array}$ & $P$ value & Effect size \\
\hline \multicolumn{6}{|c|}{ BDNF (pg/mL) } \\
\hline Exercise & $4642.51(7065.08)$ & 5308.17 (6355.73) & $123.96(2690.97)$ & 0.07 & $\mathrm{n} / \mathrm{a}$ \\
\hline Control & $5330.86(10346.65)$ & 4477.95 (5002.88) & $-530.79(1427.44)$ & & \\
\hline \multicolumn{6}{|c|}{ BDNF (pg/mL) outliers excluded } \\
\hline Exercise & $4564.61(5737.23)$ & 5173.27 (5997.54) & $135.86(1709.74)$ & $0.024^{*}$ & 0.3 \\
\hline Control & 4593.74 (9558.29) & 3974.66 (3668.22) & $-530.79(838.43)$ & & \\
\hline \multicolumn{6}{|c|}{ CANTAB PALTEA } \\
\hline Exercise & $44(30)$ & $46(23)$ & $.26(10.29)$ & 0.08 & $\mathrm{n} / \mathrm{a}$ \\
\hline Control & $44(22)$ & $33(29)$ & $-6(15.3)$ & & \\
\hline \multicolumn{6}{|c|}{ Stroop CWS } \\
\hline Exercise & $66.42(28.51)$ & $70.48(30.66)$ & $4.00(16.00)$ & 0.456 & $\mathrm{n} / \mathrm{a}$ \\
\hline Control & 75.25 (26.97) & $77.93(27.42)$ & $1.00(13.00)$ & & \\
\hline \multicolumn{6}{|c|}{ SART commission errors } \\
\hline Exercise & $2.5(5)$ & $4(4)$ & $0.5(5)$ & $0.025^{*}$ & 0.28 \\
\hline Control & $3(4)$ & $2(3)$ & $-1(3)$ & & \\
\hline \multicolumn{6}{|c|}{ SART reaction time (ms) } \\
\hline Exercise & $142.04(83.50)$ & 128.07 (74.42) & $4.84(39.24)$ & 0.055 & $\mathrm{n} / \mathrm{a}$ \\
\hline Control & $154.57(78.90)$ & $123.61(79.62)$ & $27.66(51.36)$ & & \\
\hline
\end{tabular}

Primary analysis: Between-group differences T1 and T2 time points. Data are presented for the exercise (group A) and control group (group B) as mean \pm SD for normally distributed data and as median \pm IQR for non-normally distributed data. $P$ value represents within-group differences with statistical significance.

${ }^{*} \mathrm{P}<0.05$.

BDNF, brain-derived neurotrophic factor; CWS, colour word score; PALTEA, Paired Associates Learning Total Errors Adjusted; SART, Sustained Attention to Response Task; n/a, not applicable.

participants in exercise BDNF response and other cognitive measures.

\section{DISCUSSION}

This study examined the changes in sBDNF concentration and cognitive performance in response to a short bout of high-intensity aerobic exercise in individuals with MCI. To the best of our knowledge, this is the first study to show that a short bout of high-intensity aerobic exercise increases peripheral sBDNF in a population with MCI. The results support previous work in this area which has primarily focused on young healthy cohorts. ${ }^{22} 26-33$
However, in the current study secondary analysis demonstrated only modest within-group post exercise cognitve change.

The findings of the present study are consistent with previous studies that have reported increases in peripheral BDNF in response to acute exercise protocols in healthy adult cohorts ${ }^{22} 26-33$ and in clinical populations. ${ }^{34} 35$ To date, few studies have examined the effect of acute aerobic exercise on peripheral BDNF in older adults $^{36-40}$ or MCI. ${ }^{41}$ However, a recent study by Tsai $e t$ $a l^{41}$ found an acute bout of 30 min of moderate-intensity aerobic exercise significantly increased sBDNF levels

Table 4 Secondary analysis measuring change over time in study outcomes within group B

\begin{tabular}{lllll}
\hline Variables & Baseline T1 & Postcontrol condition T2 & Postexercise condition T3 & P value \\
\hline BDNF $(\mathrm{pg} / \mathrm{mL})$ outliers excluded & $4593.74(9558.29)$ & $3974.66(3668.22)$ & $4790.90(6713.17)$ & $0.005^{\star}$ \\
CANTAB PALTEA & $44(22)$ & $33(29)$ & $28(30)$ & 0.03 \\
Stroop CWS & $75.25(26.97)$ & $77.93(27.42)$ & $83.93(21.47)$ & $0.005^{\star}$ \\
SART commission errors & $3(4)$ & $2(3)$ & $2(3)$ & 0.25 \\
SART reaction time $(\mathrm{ms})$ & $154.57(78.90)$ & $123.61(79.62)$ & $142.07(65.87)$ & $0.005^{\star}$ \\
\hline
\end{tabular}

$P$ value represents within group change over time with statistical significance.

Bonferroni correction applied and alpha level ${ }^{*} \mathrm{P}<0.025$.

BDNF, brain-derived neurotrophic factor; CWS, colour word score; PALTEA, Paired Associates Learning Total Errors Adjusted; SART,

Sustained Attention to Response Task. 
in a group $(n=25)$ of older adults with amnestic MCI. In agreement, our findings indicate that older adults with MCI, despite being in the early stages of the neurodegenerative process, retain the ability to increase peripheral BDNF concentration following acute aerobic exercise of varying intensity and duration.

Across the literature, the $\% \mathrm{BDNF}$ change in response to exercise varies widely in healthy and clinical populations, with an average increase of $60 \%$ reported in peripheral BDNF levels following acute exercise. ${ }^{23} 42$ The magnitude of BDNF change in response to exercise is dependent on blood lactate concentration, duration and intensity of exercise and is transient in nature. ${ }^{2324263143-45}$ The within-group sBDNF change in response to exercise observed in our results was $7.07 \%$ (group A) and $15.7 \%$ (group B). Studies of similar study design that examined comparable graded exercise protocols in healthy young cohorts $^{222627}$ and clinical populations ${ }^{3646}$ reported higher $\%$ BDNF change in response to exercise $(16 \%-30 \%$ relative change from baseline). Coelho $e t a l^{36}$ examined the exercise BDNF response in 21 older adults with $\mathrm{AD}$ and 18 healthy older adults and found significant increases in BDNF plasma levels in both groups ( $\mathrm{p}=0.001 ; F=13.63$; $\mathrm{df}=37$ ) with a relative change of $22 \%$ and $16 \%$, respectively. Despite comparable performance of the exercise condition (duration and intensity) in both groups, group B demonstrated a higher \% increase in sBDNF after exercise. In contrast to existing literature, the BDNF upregulation did not appear to be driven by lactate ${ }^{45}$ but may be explained by the significantly higher CR fitness observed in group B.

CR fitness in the present study cohort was low, with $n=59$ $(92.2 \%)$ of participants categorised as very poor $(<20$ th percentile) or poor (20th-40th percentiles).$^{25}$ While evidence supports strong associations between higher levels of CR fitness and cognitive function, ${ }^{47}$ the relationship between BDNF and CR fitness is unclear, with some studies reporting a positive association between the two immediately after exercise ${ }^{49}$ and others reporting an inverse relationship. ${ }^{50-52} \mathrm{~A}$ meta-analytical review by Dinoff $e t a l^{42}$ reported significant associations between $\mathrm{VO}_{2}$ peak and effect sizes in studies measuring the effects of acute exercise on BDNF, indicating greater increases in peripheral BDNF after acute exercise in those with greater CR fitness. In the present study, we could not compare BDNF response in high and low-fit participants due to the homogeneity in the study sample but no correlation was found between CR fitness $\left(\mathrm{VO}_{2} \max \right)$ and baseline sBDNF levels.

Despite the consistently reported positive effects of acute exercise on cognitive performance, ${ }^{14} 28$ 53-55 the findings of the current study do not support the beneficial effect of acute exercise on cognitive performance in MCI. In contrast, the resting control condition demonstrated a decrease in sBDNF and improved performance on the SART, a task that activates the cortical and subcortical attentional networks. ${ }^{56}$ While it is possible that the resting control condition may have reduced circulating cortisol levels similar to quiet meditation practice, ${ }^{5758}$ the mechanisms underpinning the observed effect cannot be fully explained with further research required.

Recent meta-analytical reviews report that the BDNF and cognitive response to exercise may vary in a sex-dependent manner. ${ }^{42}{ }^{59}$ Subgroup analysis by Dinoff $e t a t^{42}$ revealed significant increase in BDNF after acute exercise in males but not in females. In contrast, our findings did not reveal sex differences in BDNF response to acute exercise. However, we did observe sex differences in SART RT with females gaining significant improvement in RT following acute exercise compared with males. RTs are known to slow and become more variable with age, with significant sex differences reported in RT variability. ${ }^{60}$ Despite recent reports of sex differences in the cognitive response in executive function tasks to exercise ${ }^{59}$ we did not observe any sex differences in other cognitive measures in response to acute exercise.

The results of the present study should be viewed in the context of a number of potential limitations. First, peripheral measurement of BDNF is not without limitations, as processes occurring centrally in the brain may not have a measurable influence on peripheral blood markers; nor may circulating BDNF have any influence on brain function within the time frame of this experiment. Second, secondary analysis of change in cognitive performance (group B) demonstrated improvement in executive function after exercise. However, group B completed an additional measurement of cognitive measures (T3), thereby increasing the risk of type I error due to practice effects of repeated tests which may be an important confounder of these results. ${ }^{61}$ Finally, other factors that may mediate BDNF response to exercise were not examined. The BDNF val66met polymorphism, a genetic variant that occurs in $33 \%$ of the population, and the apolipoprotein $\mathrm{E}$ gene $\varepsilon 4$ allele, the most prevalent genetic risk factor for late-onset $\mathrm{AD}$, affect the expression of BDNF, ${ }^{62}$ alter susceptibility to neuropathology and may be an important consideration in understanding responders to exercise. ${ }^{63-67}$

\section{CONCLUSIONS}

The results of this study indicate that a single session of high-intensity exercise is feasible in an MCI cohort, enhances circulating sBDNF concentration but does not improve visuospatial learning and memory, sustained attention or executive function. This research adds to the literature which postulates that the impaired BDNF profile in MCI cohorts may be amenable to exercise training as a strategy to address the declining BDNF profile.

Acknowledgements We acknowledge the assistance and support of the Wellcome Trust-HRB Clinical Research Facility at St James' Hospital in providing a dedicated environment for the conduct of high-quality clinical research activities.

Collaborators Members of the NeuroExercise Study Group (in alphabetical order by name): Dr. Justine A. Aaronson, Department of Medical Psychology, Radboud University Medical Center, Nijmegen, The Netherlands; Dr. Vera Abeln, Institute of Movement and Neurosciences, German Sport University Cologne, Germany; Dr. 
Jurgen A.H.R Claassen, Department of Geriatric Medicine, Radboud University Medical Center, Donders Institute for Brain, Cognition and Behaviour, Nijmegen, The Netherlands; Dr. Robert F. Coen, Mercer's Institute for Research on Ageing, St. James's Hospital, Dublin, Ireland; Dr. Damien Ferguson, Department of Neurology, St. James's Hospital, Dublin, Ireland; Prof. Roy P.C. Kessels, Department of Medical Psychology, Radboud University Medical Center, Donders Institute for Brain, Cognition and Behaviour, Nijmegen, The Netherlands; Prof. Romain Meeusen, Human Physiology Research Group, Vrije Universiteit Brussels, Belgium; Prof. Christian Montag, Institute of Psychology and Education, Ulm University, Germany and Key Laboratory for Neurolnformation/Center for Information in Medicine, and School of Life Science and Technology, University of Electronic Science and Technology of China, Chengdu, China; Dr Ross T. Murphy, St. James's Hospital, Dublin, Ireland; Prof. M. Cristina Polidori, Ageing Clinical Research, University Hospital of Cologne, Germany; Prof. Martin Reuter, University of Bonn, Department of Psychology and Center for Economics \& Neuroscience, Laboratory of Neurogenetics, Bonn, Germany; Marit Sanders, Department of Geriatric Medicine, Radboud Alzheimer Centre, Radboud University Medical Center, Nijmegen, The Netherlands;Prof. Heiko K. Strüder, Institute of Movement and Neurosciences, German Sport University Cologne, Germany; Tim Stuckenschneider, Institute of Movement and Neurosciences, German Sport University Cologne, Germany; Prof H.J. Thijssen, Department of Physiology, Radboud University Medical Center, Nijmegen, The Netherlands; Prof. Tobias Vogt, Institute for Professional Sport Education and Sport Qualifications, German Sport University Cologne, Germany; Prof. Bernd Weber, Department of Epileptology, University Hospital Bonn, Germany.

Contributors KED, EMG, BL, MOR and SS were involved in study conception and design. AK and BCM completed the laboratory analysis and assisted with BDNF analysis. CW advised and assisted on statistical analysis. KED and EMG drafted the manuscript with input from all authors on each version of the paper. All authors have approved the final version of this paper prior to submission.

Funding This study is part of the NeuroExercise project which has been supported by The EU Joint Programme-Neurodegenerative Disease Research (JPND). Local funding was provided through the Health Research Board, Ireland.

Disclaimer The sponsors had no involvement in the study design, collection, analysis and interpretation of data or in writing of the report or decision to submit the paper for publication.

Competing interests None declared.

Patient consent for publication Obtained.

Ethics approval Research and Ethics Committees (REC): Tallaght Hospital/ St James' Hospital Joint REC, Mater Misericordiae University Hospital REC, St Patrick's University Hospital REC and St Vincent's University Hospital REC.

Provenance and peer review Not commissioned; externally peer reviewed.

Data sharing statement Data are available upon reasonable request.

Open access This is an open access article distributed in accordance with the Creative Commons Attribution Non Commercial (CC BY-NC 4.0) license, which permits others to distribute, remix, adapt, build upon this work non-commercially, and license their derivative works on different terms, provided the original work is properly cited, appropriate credit is given, any changes made indicated, and the use is non-commercial. See: http://creativecommons.org/licenses/by-nc/4.0/.

\section{REFERENCES}

1. Roberts R, Knopman DS. Classification and epidemiology of $\mathrm{MCl}$ Clin Geriatr Med 2013;29:753-72.

2. Farias ST, Mungas D, Reed BR, et al. Progression of mild cognitive impairment to dementia in Clinic- vs community-based cohorts. Arch Neurol 2009;66:1151-7.

3. Petersen RC, Lopez O, Armstrong MJ, et al. Practice guideline update summary: mild cognitive impairment: report of the Guideline Development, Dissemination, and Implementation Subcommittee of the American Academy of Neurology. Neurology 2018;90:126-35.

4. Chang YK, Labban JD, Gapin JI, et al. The effects of acute exercise on cognitive performance: a meta-analysis. Brain Research 2012:1453:87-101.

5. Lambourne K, Tomporowski P. The effect of exercise-induced arousal on cognitive task performance: a meta-regression analysis. Brain Research 2010;1341:12-24.

6. McMorris T, Hale BJ. Differential effects of differing intensities of acute exercise on speed and accuracy of cognition: a metaanalytical investigation. Brain and Cognition 2012;80:338-51.
7. Egan MF, Kojima M, Callicott JH, et al. The BDNF Val66Met polymorphism affects activity-dependent secretion of BDNF and human memory and hippocampal function. Cell 2003;112:257-69

8. Lommatzsch M, Zingler D, Schuhbaeck K, et al. The impact of age, weight and gender on BDNF levels in human platelets and plasma. Neurobiology of Aging 2005;26:115-23.

9. Ziegenhorn AA, Schulte-Herbrüggen $\mathrm{O}$, Danker-Hopfe $\mathrm{H}$, et al. Serum neurotrophins-A study on the time course and influencing factors in a large old age sample. Neurobiology of Aging 2007;28:1436-45.

10. Forlenza OV, Miranda AS, Guimar I, et al. Decreased neurotrophic support is associated with cognitive decline in non-demented subjects. Journal of Alzheimer's Disease 2015;46:423-9.

11. Peng S, Wuu J, Mufson EJ, et al. Precursor form of brain-derived neurotrophic factor and mature brain-derived neurotrophic factor are decreased in the pre-clinical stages of Alzheimer's disease. Journal of Neurochemistry 2005;93:1412-21.

12. Shimada H, Makizako H, Doi T, et al. A large, cross-sectional observational study of serum BDNF, cognitive function, and mild cognitive impairment in the elderly. Frontiers in Aging Neuroscience 2014;6(Suppl. 1).

13. Basso JC, Suzuki WA. The Effects of Acute Exercise on Mood, Cognition, Neurophysiology, and Neurochemical Pathways: A Review. Brain Plast 2017;2:127-52.

14. Piepmeier AT, Etnier JL. Brain-derived neurotrophic factor (BDNF) as a potential mechanism of the effects of acute exercise on cognitive performance. J Sport Health Sci 2015;4:14-23.

15. Sanders ML, Stuckenschneider T, Devenney KE, et al. Real world recruiting of older subjects with mild cognitive impairment for exercise trials: community readiness is pivotal. Journal of Alzheimer's Disease 2018;62:579-81.

16. Devenney KE, Sanders ML, Lawlor B, et al. The effects of an extensive exercise programme on the progression of mild cognitive impairment $(\mathrm{MCl})$ : study protocol for a randomised controlled trial. BMC Geriatrics 2017:17.

17. Albert MS, DeKosky ST, Dickson D, et al. The diagnosis of mild cognitive impairment due to Alzheimer's disease: Recommendations from the National Institute on Aging-Alzheimer's Association workgroups on diagnostic guidelines for Alzheimer's disease. Alzheimer's \& Dementia 2011;7:270-9.

18. Nasreddine ZS, Phillips NA, BÃ@dirian ValÃ@rie, et al. The Montreal Cognitive assessment, MoCA: a brief screening tool for mild cognitive impairment. Journal of the American Geriatrics Society 2005;53:695-9.

19. Strauss E, Sherman EM, Spreen O. A compendium of neuropsychological tests: Administration, norms. and commentary: American Chemical Society, 2006.

20. Fletcher GF, Balady GJ, Amsterdam EA, et al. Exercise standards for testing and training: a statement for healthcare professionals from the American Heart Association. Circulation 2001:104:1694-740.

21. Szuhany KL, Bugatti M, Otto MW. A meta-analytic review of the effects of exercise on brain-derived neurotrophic factor. Journal of Psychiatric Research 2015;60:56-64.

22. Griffin Éadaoin W, Mullally S, Foley $\mathrm{C}$, et al. Aerobic exercise improves hippocampal function and increases BDNF in the serum of young adult males. Physiol Behav 2011;104:934-41.

23. Knaepen K, Goekint M, Heyman EM, et al. Neuroplasticity exercise-induced response of peripheral brain-derived neurotrophic factor. Sports Medicine 2010;40:765-801

24. Saucedo Marquez CM, Vanaudenaerde B, Troosters T, et al. Highintensity interval training evokes larger serum BDNF levels compared with intense continuous exercise. Journal of Applied Physiology 2015;119:1363-73.

25. Medicine ACoS. ACSM's guidelines for exercise testing and prescription. Lippincott Williams \& Wilkins, 2013.

26. Ferris LEET, Williams JS, Shen C-LI. The effect of acute exercise on serum brain-derived neurotrophic factor levels and cognitive function. Medicine \& Science in Sports \& Exercise 2007;39:728-34.

27. Rojas Vega S, Strüder HK, Vera Wahrmann B, et al. Acute BDNF and cortisol response to low intensity exercise and following ramp incremental exercise to exhaustion in humans. Brain Research 2006;1121:59-65.

28. Winter B, Breitenstein C, Mooren FC, et al. High impact running improves learning. Neurobiology of Learning and Memory 2007:87:597-609.

29. Tsai C-L, Wang C-H, Pan C-Y, et al. Executive function and endocrinological responses to acute resistance exercise. Frontiers in Behavioral Neuroscience 2014;8. 
30. Tonoli C, Heyman E, Roelands B, et al. BDNF, IGF-I, glucose and insulin during continuous and interval exercise in type 1 diabetes. International Journal of Sports Medicine 2015;36:955-9.

31. Tang SW, Chu E, Hui T, et al. Influence of exercise on serum brain-derived neurotrophic factor concentrations in healthy human subjects. Neuroscience Letters 2008;431:62-5.

32. Hwang J, Brothers RM, Castelli DM, et al. Acute high-intensity exercise-induced cognitive enhancement and brain-derived neurotrophic factor in young, healthy adults. Neuroscience Letters 2016;630:247-53

33. Hötting K, Schickert N, Kaiser J, et al. The effects of acute physical exercise on memory, peripheral BDNF, and cortisol in young adults. Neural Plasticity 2016;2016:1-12.

34. Gustafsson G, Lira CM, Johansson J, et al. The acute response of plasma brain-derived neurotrophic factor as a result of exercise in major depressive disorder. Psychiatry Research 2009;169:244-8.

35. Castellano V, White LJ. Serum brain-derived neurotrophic factor response to aerobic exercise in multiple sclerosis. Journal of the Neurological Sciences 2008;269:85-91.

36. Coelho FGdeM, Vital TM, Stein AM, et al. Acute aerobic exercise increases brain-derived neurotrophic factor levels in elderly with Alzheimer's disease. J Alzheimers Dis 2014;39:401-8.

37. Gomes WF, Lacerda ACR, Mendonça VA, et al. Effect of exercise on the plasma BDNF levels in elderly women with knee osteoarthritis. Rheumatology International 2014;34:841-6.

38. Babaei P, Damirchi A, Mehdipoor M, et al. Long term habitual exercise is associated with lower resting level of serum BDNF. Neuroscience Letters 2014;566:304-8.

39. Dimitrova J, Hogan M, Khader P, et al. Comparing the effects of an acute bout of physical exercise with an acute bout of interactive mental and physical exercise on electrophysiology and executive functioning in younger and older adults. Aging Clinical and Experimental Research 2017;29:959-67.

40. Håkansson K, Ledreux A, Daffner K, et al. BDNF Responses in Healthy Older Persons to 35 Minutes of Physical Exercise, Cognitive Training, and Mindfulness: Associations with Working Memory Function. Journal of Alzheimer's Disease 2017;55:645-57.

41. Tsai C-L, Ukropec J, Ukropcová B, et al. An acute bout of aerobic or strength exercise specifically modifies circulating exerkine levels and neurocognitive functions in elderly individuals with mild cognitive impairment. Neurolmage: Clinical 2018;17:272-84.

42. Dinoff A, Herrmann N, Swardfager W, et al. The effect of acute exercise on blood concentrations of brain-derived neurotrophic factor in healthy adults: a meta-analysis. European Journal of Neuroscience 2017;46:1635-46.

43. Schmidt-Kassow M, Zink N, Mock J, et al. Treadmill walking during vocabulary encoding improves verbal long-term memory. Behavioral and Brain Functions 2014;10.

44. Schmolesky MT, Webb DL, Hansen RA. The effects of aerobic exercise intensity and duration on levels of brain-derived neurotrophic factor in healthy men. J Sports Sci Med 2013;12.

45. Schiffer T, Schulte S, Sperlich B, et al. Lactate infusion at rest increases BDNF blood concentration in humans. Neuroscience Letters 2011;488:234-7.

46. Laske C, Banschbach S, Stransky E, et al. Exercise-induced normalization of decreased BDNF serum concentration in elderly women with remitted major depression. Int. J. Neuropsychopharm. 2010;13:595-602.

47. Wong CN, Chaddock-Heyman L, Voss MW, et al. Brain activation during Dual-task processing is associated with cardiorespiratory fitness and performance in older adults. Frontiers in Aging Neuroscience 2015;7.

48. Smith PJ, Blumenthal JA, Hoffman BM, et al. Aerobic exercise and neurocognitive performance: a meta-analytic review of randomized controlled trials. Psychosomatic Medicine 2010;72:239-52.
49. Cho H-C, Kim J, Kim S, et al. The concentrations of serum, plasma and platelet $\mathrm{BDNF}$ are all increased by treadmill $\mathrm{VO}_{2} \max$ performance in healthy College men. Neurosci Lett 2012;519:78-83.

50. Tomporowski PD. Effects of acute bouts of exercise on cognition. Acta Psychologica 2003;112:297-324.

51. Jung SH, Kim J, Davis JM, et al. Association among basal serum BDNF, cardiorespiratory fitness and cardiovascular disease risk factors in untrained healthy Korean men. European Journal of Applied Physiology 2011;111:303-11.

52. Currie J, Ramsbottom R, Ludlow $\mathrm{H}$, et al. Cardio-respiratory fitness, habitual physical activity and serum brain derived neurotrophic factor (BDNF) in men and women. Neuroscience Letters 2009:451:152-5.

53. Skriver K, Roig M, Lundbye-Jensen J, et al. Acute exercise improves motor memory: exploring potential biomarkers. Neurobiology of Learning and Memory 2014;116:46-58.

54. Basso JC, Shang A, Elman M, et al. Acute exercise improves prefrontal cortex but not hippocampal function in healthy adults. Journal of the International Neuropsychological Society 2015;21:791-801.

55. Hillman $\mathrm{CH}$, Snook EM, Jerome GJ. Acute cardiovascular exercise and executive control function. International Journal of Psychophysiology 2003:48:307-14.

56. O'Connor C, Robertson IH, Levine B. The prosthetics of vigilant attention: random cuing cuts processing demands. Neuropsychology 2011;25:535-43.

57. O'Leary K, O'Neill S, Dockray S. A systematic review of the effects of mindfulness interventions on cortisol. Journal of Health Psychology 2016;21:2108-21.

58. Fox KCR, Nijeboer S, Dixon ML, et al. Is meditation associated with altered brain structure? A systematic review and meta-analysis of morphometric neuroimaging in meditation practitioners. Neurosci Biobehav Rev 2014;43:48-73.

59. Barha CK, Davis JC, Falck RS, et al. Sex differences in exercise efficacy to improve cognition: a systematic review and metaanalysis of randomized controlled trials in older humans. Frontiers in Neuroendocrinology 2017:46:71-85.

60. Der G, Deary IJ. Age and sex differences in reaction time in adulthood: results from the United Kingdom health and lifestyle survey. Psychology and Aging 2006;21:62-73.

61. Goldberg TE, Harvey PD, Wesnes KA, et al. Practice effects due to serial cognitive assessment: implications for preclinical Alzheimer's disease randomized controlled trials. Alzheimers Dement 2015;1:103-11.

62. Allard JS, Ntekim O, Johnson SP, et al. APOE\&4 impacts upregulation of brain-derived neurotrophic factor after a six-month stretch and aerobic exercise intervention in mild cognitively impaired elderly African Americans: a pilot study. Experimental Gerontology 2017;87:129-36

63. Leckie RL, Oberlin LE, Voss MW, et al. BDNF mediates improvements in executive function following a 1-year exercise intervention. Frontiers in Human Neuroscience 2014;8.

64. Erickson Kl, Banducci SE, Weinstein AM, et al. The brain-derived neurotrophic factor Val66Met polymorphism moderates an effect of physical activity on working memory performance. Psychological Science 2013;24:1770-9.

65. Watts A, Andrews SJ, Anstey KJ. Sex Differences in the Impact of BDNFGenotype on the Longitudinal Relationship between Physical Activity and Cognitive Performance. Gerontology 2018:64:361-72.

66. Hopkins ME, Davis FC, VanTieghem MR, et al. Differential effects of acute and regular physical exercise on cognition and affect. Neuroscience 2012;215:59-68.

67. Nascimento CMC, Pereira JR, Pires de Andrade L, et al. Physical exercise improves peripheral BDNF levels and cognitive functions in mild cognitive impairment elderly with different BDNF Val66Met genotypes. Journal of Alzheimer's Disease 2014:43:81-91. 